\title{
A full-thickness skin construct made of a collagen hydrogel strengthened by a fibrin-modified nanofibrous membrane
}

\author{
Julia Pajorova ${ }^{1,2}$, Marketa Bacakova ${ }^{1}$, Antonin Broz ${ }^{1}$, Martina Travnickova ${ }^{1,2}$, Lucie Bacakova ${ }^{1}$ \\ ${ }^{1}$ Dept. of Biomaterials and Tissue Engineering, Institute of Physiology CAS, Prague, Czech Republic \\ ${ }^{2}$ Second Faculty of Medicine, Charles University, Prague, Czech Republic
}

INSTITUIF

\section{Introduction}

For many years, a three-dimensional (3D) collagen hydrogel has been used for embedding the fibroblasts to provide them with physiological 3D environment. However, the stiffness of tissueengineered skin analogues based on collagen hydrogels is insufficient. The cell traction forces typically cause the shrinkage of the hydrogels. In order to improve the mechanical properties of a two-layer skin construct, we stabilized the collagen hydrogel by fibrin-modified nanofibrous membrane.

\section{Materials \& Methods}

\begin{abstract}
Nanofibrous polylactid acid (PLLA) membranes were modified with fibrin homogenous mesh. Fibrinmodified membranes were seeded with human dermal fibroblasts (FIB) or adipose-derived stem cells (ASC). After few days, collagen hydrogel was applied on the surface of fibrin-modified membranes pre-seeded with fibroblasts or stem cells, and then primary keratinocytes (KERAT) were seeded on the surface of collagen hydrogel. Cell proliferation, migration and morphology were evaluated by super-resolution microscopy.
\end{abstract}

\section{Results}

\section{Fibroblasts on fibrin-coated PLLA membrane}
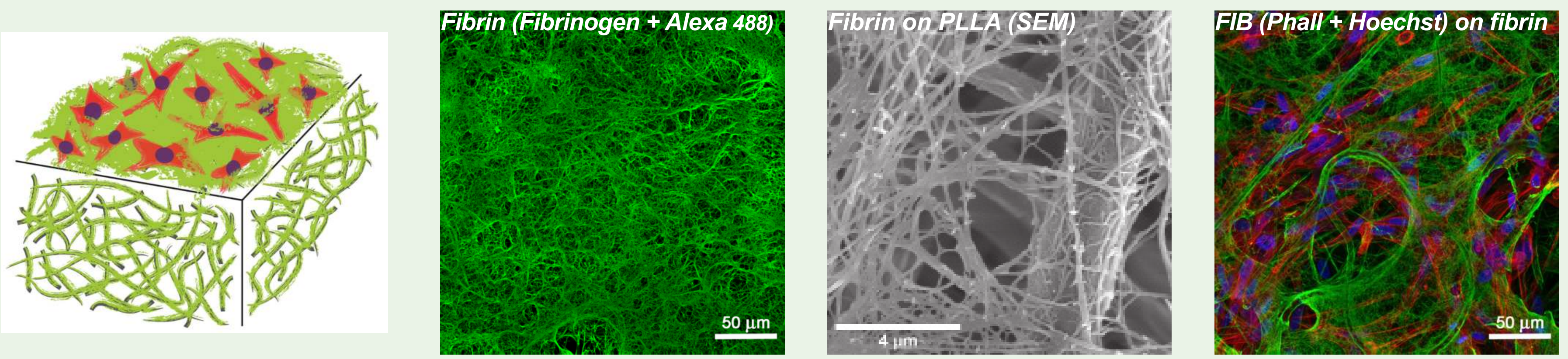

2. Fibroblasts migrated into collagen gel from fibrin-modified PLLA membrane
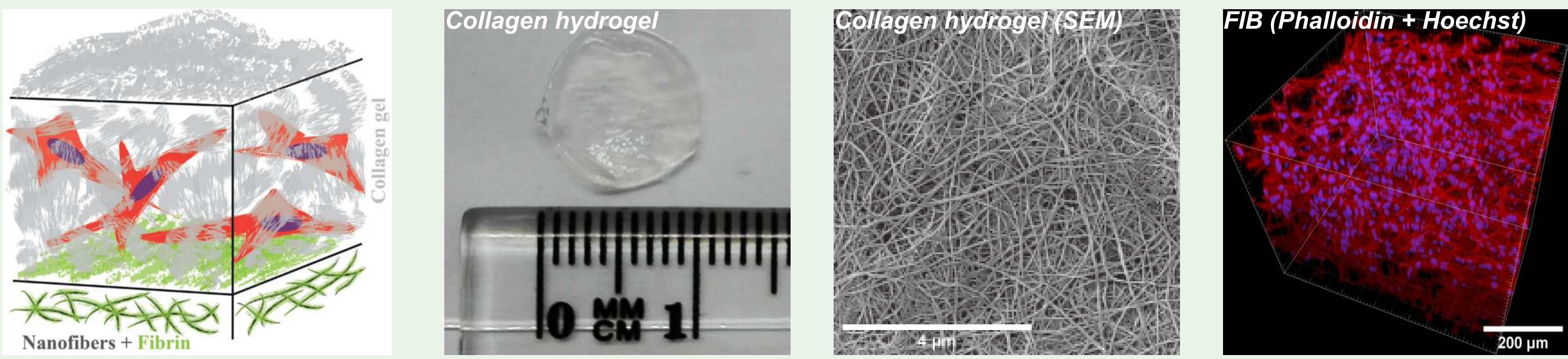

3. Bilayered construct of fibroblasts and keratinocytes strengthened by fibrin-modified membrane
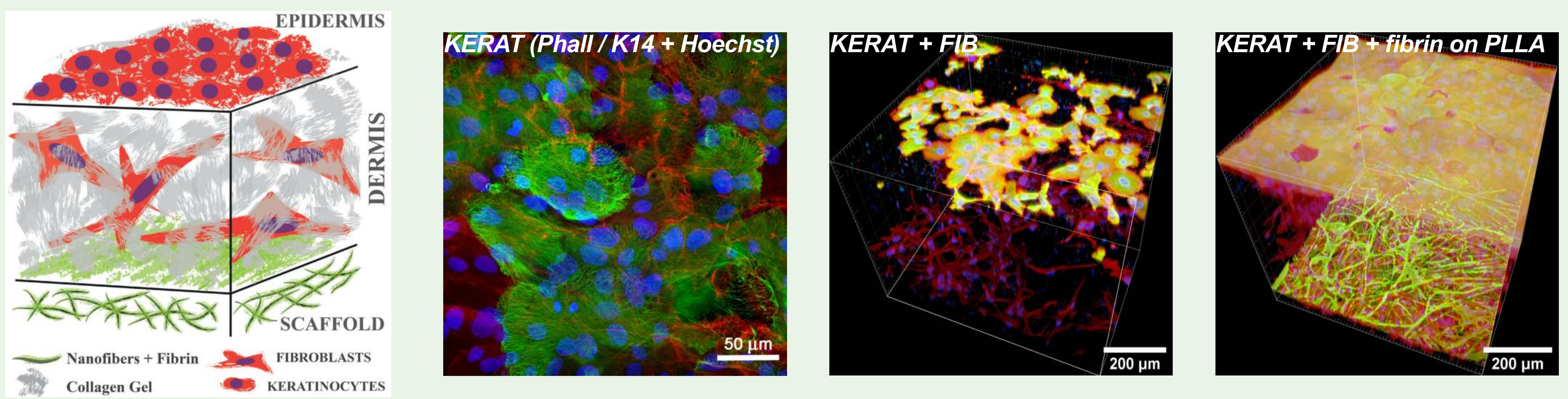